\title{
GANGRENA DE FOURNIER
}

FOURNIER'S GANGRENE

João B Cardoso', Omar Féres²

${ }^{1}$ Médico Assistente. Disciplina de Cirurgia de Urgência e Trauma. ${ }^{2}$ Docente. Disciplina de Proctologia. Departamento de Cirurgia e Anatomia. Faculdade de Medicina de Ribeirão Preto - USP.

CorRespondência: Centro de Estudos de Emergências em Saúde. Rua Bernardino de Campos, 1000 - 2ํandar. 14015-030 Ribeirão Preto - SP. e-mail: jobacar@yahoo.com

Cardoso JB, Féres O. Gangrena de Fournier. Medicina (Ribeirão Preto) 2007; 40 (4): 493-9, out./dez.

RESUMO: A Gangrena de Fournier é uma grave infecção polimicrobiana que evolui com fasciite necrotizante, comprometendo principalmente as regiões genital, perineal e perianal. Caracteriza-se por rápida evolução e pode complicar com sepse, falência de múltiplos órgãos e óbito. A base do tratamento é o diagnóstico precoce e o desbridamento agressivo. Antibioticoterapia de amplo espectro, oxigenoterapia hiperbárica e cuidados locais são medidas complementares.

Descritores: Gangrena de Fournier. Infecção. Fasciite Necrosante. Sepse.

\section{1- INTRODUÇÃO}

A Gangrena de Fournier é uma infecção polimicrobiana causada por microorganismos aeróbios e anaeróbios que, atuando de maneira sinérgica, determinam um fasciite necrotizante acometendo principalmente as regiões genital, perineal e perianal. ${ }^{1}$

Esse processo infeccioso através de uma endarterite obliterante leva à trombose dos vasos cutâneos e subcutâneos e conseqüente necrose da pele da região acometida. ${ }^{1,2,3}$

Sem tratamento, o processo pode não só estender-se rapidamente à parede abdominal anterior, à região dorsal, aos membros superiores e ao retroperitônio bem como induzir à sepse, à falência de múltiplos órgãos e à morte.

Relatada pela primeira vez em 1764 por Baurienne e referida na literatura com uma rica sinonímia recebeu o nome de Gangrena de Fournier em homenagem ao urologista francês Jean Alfred Fournier que a descreveu com detalhes em dois trabalhos publicados em 1863 e $1864 .^{4,5,6}$
Considerada inicialmente um processo limitado ao sexo masculino, idiopático e fulminante em sua descrição original hoje é uma entidade bem conhecida, mas que ainda persiste com índices variáveis, porém elevados de mortalidade, alcançando em algumas séries $40 \%$ a $67 \% \%^{3,7,8,9}$

\section{2- EPIDEMIOLOGIA}

Embora originariamente descrita como uma doença que afeta o indivíduo jovem do sexo masculino, teve esse perfil mudado em descrições mais recentes. $^{3}$

A doença, embora ocorra principalmente em indivíduos do sexo masculino na proporção de 10 para 1, não é restrita aos indivíduos jovens, afetando todas as faixas etárias, com média das idades ao redor dos 50 anos. $3,10,11$

É uma doença relativamente incomum. Até 1984 estimava-se em 300 o número de casos descritos na literatura. ${ }^{12}$

Contudo, tem sido motivo de publicações cons- 
tantes quer em artigos de revisão ${ }^{11}$, quer como relato de $\operatorname{casos}^{13 / 16}$ quer como estudos de casuísti$\operatorname{cas}^{2,3,7,10,11,17 / 24}$ das quais, uma das maiores, foi publicada, em nosso meio, por Féres et al. que estudaram 88 casos em um período de 10 anos. ${ }^{17}$

Algumas doenças sistêmicas parecem ser fatores de risco, para o desenvolvimento da gangrena, entre estas o diabetes mellitus encontrado em 40 a $60 \%$ dos pacientes, o alcoolismo encontrado em 25 a $50 \%$ dos casos, a hipertensão arterial, a obesidade, o tabagismo e as doenças e condições imunossupressoras como infecção pelo HIV, radio e quimioterapia, leucemias, dentre outras. ${ }^{2,9,10,17}$

Face ao número crescente de casos de infecção pelo HIV na população alguns autores têm sugerido que a Gangrena de Fournier possa ser a primeira manifestação de infecção pelo HIV recomendando a pesquisa dessa infecção em pacientes que desenvolvem a gangrena sem um fator causal aparente.,24

\section{3- ETIOLOGIA}

Inicialmente descrita como uma doença de causa desconhecida, sabe-se hoje que um processo patológico subjacente pode ser encontrado na maioria dos casos de Gangrena de Fournier embora ainda em um número expressivo dos pacientes a causa não possa ser determinada. $3,9,10,11,25$

Portanto uma investigação cuidadosa pode demonstrar a porta de entrada que pode ser localizada, sobretudo, no trato urogenital, no trato digestivo ou em afecções cutâneas. ${ }^{11,12,25}$

Focos no trato urogenital, descritos na literatura, incluem causas diversas: estenoses uretrais, son- dagem vesical de demora, abscesso escrotal, orquites, epididimites, abscesso renal, cateterização uretral traumática, cálculos uretrais, cálculos vesicais, câncer vesical, câncer de pênis, massagem prostática e biópsia prostática. $2,3,8,10,26$

No trato digestório foram relatados focos originários em abscessos perianais, carcinomas do cólon e do reto, apendicites e diverticulites agudas, Doença de Crohn, hérnias encarceradas, e perfuração do reto por corpo estranho (osso de frango). ${ }^{11,19,25,27}$

Em artigo de revisão, abrangendo 1726 casos, as afecções cutâneas lideram a sede dos focos de infecção respondendo por $24 \%$ dos casos. ${ }^{11}$

Entre estas se destacam as bartolinites, abscessos subcutâneos superficiais e complicações de procedimentos cirúrgicos comuns e de porte relativamente pequeno como vasectomia, hemorroidectomia, orquiectomia, herniorrafia, cirurgia para correção de hidrocele e postectomia ${ }^{3,16}$ (Tabela I).

\section{4- MICROBIOLOGIA}

Diversos estudos demonstram que a Gangrena de Fournier é uma infecção polimicrobiana, um verdadeiro caldo de cultura onde são encontradas bactérias aeróbias e anaeróbias que habitualmente não são patogênicas, mas que associadas, e em condições favoráveis, são devastadoras. ${ }^{2,3,7,8,10,11,25,28}$

As culturas realizadas demonstram, em média, a presença de quatro microorganismos por paciente ${ }^{2}$.

Os microrganismos mais freqüentemente isolados entre os Gram negativos aeróbios são Escherichia coli, Klebsiella pneumoniae, Pseudomonas aeruginosa e Proteus mirabilis. , $3,7,8,10,11,20,25_{\text {En- }}$

Tabela I: Principais etiologias e locais de origem da Gangrena de Fournier. 2,3,8,10,11,16,25,26,27

\begin{tabular}{lll}
\hline Trato digestório e anoretal & Urogenital & Cutâneo \\
\hline Abscesso perianal & Infecção periuretral & Trauma \\
Carcinoma colo-retal & Estenose uretral & Vasectomia \\
Apendicite & Câncer de bexiga & Orquiectomia \\
Diverticulite & Orquiepididimite & Correção de hidrocele \\
Perfuração retal & Biópsia de próstata & Abscesso superficial de pele \\
Hemorroidectomia & Cálculo uretral & Herniorrafia \\
Doença de Crohn & Cateterização uretral & Abscessos de Bartholin \\
Biópsia retal & Extravasamento peri-uretral de urina & Circuncisão \\
& & Implante de prótese peniana \\
\hline
\end{tabular}


tre os aeróbios Gram positivos destacam-se o Staphylococcus aureus, o Staphylococcus epidermidis, Streptococcus viridans e o Streptococcus fecalis. 2,3,7,8,10,11,20,25 Os anaeróbios estão representados pelo Bacteróides fragilis, Bacteróides melaninogenicus, cocos Gram positivos e Clostridium species (não perfringens). ${ }^{2,3,7,8,10,11,20,25}$

Atuando de maneira sinérgica essas bactérias agem através de diferentes mecanismos contribuindo não só para a gravidade mas também, para a rápida disseminação do processo.

Assim, a oclusão vascular é facilitada pela ação de bactérias aeróbicas por agregação plaquetária, de bactérias anaeróbias pela produção de heparinase e pela ação indutora de trombose pela ação da endotoxina de bactérias Gram-negativas. ${ }^{2,25}$

Por outro lado a destruição tissular é promovida pela ação direta da hialuronidase, produzida por estreptococos, estafilococos e bacteróides, sobre o tecido conjuntivo. ${ }^{2,25}$

A ação de outras enzimas como estreptoquinases e estreptodornases produzidas por estreptococos agravam ainda mais a destruição tissular. ${ }^{2,25}$

Ao inibir a fagocitose os bacteróides impedem a destruição de microrganismos aeróbicos. A crepitação é produzida pela produção de hidrogênio e nitrogênio por anaeróbios. ${ }^{2}$

\section{5- QUADRO CLÍNICO}

As manifestações mais freqüentes são dor, eritema, edema e necrose do escroto ou região perianal e perineal em associação com febre e calafrios ${ }^{2,3,11}$. Por outro lado, a doença pode manifestar-se de maneira insidiiosa ou como sepse. ${ }^{15}$

Outras manifestações locais incluem flictenas, crepitação, cianose e secreção com forte e repulsivo odor fétido. ${ }^{2,3,11,20}$

Deve-se lembrar que as manifestações cutâneas constituem a "ponta do iceberg" uma vez que a infecção alastra-se rápida e agressivamente ao longo de planos fasciais profundos. ${ }^{3}$

A falta de resposta ao tratamento adequado de uma celulite é forte indício da presença da doença. ${ }^{2}$

A suspeita clínica deve ser levantada quando essas manifestações aparecem em pacientes portadores de condições predisponentes para instalação da doença: diabetes, alcoolismo e imunodepressão lembrando que a Gangrena de Fournier pode ser a primeira manifestação da infecção pelo HIV ou quando as manifestações sistêmicas (febre, toxemia, prostração, taquicardia, dentre outros ) são desproporcionais às manifestações locais., ${ }^{2,3,25}$ Pela localização mais freqüente, são várias as possibilidades no diagnóstico diferencial: celulite, hérnia estrangulada, abscesso de escroto, fasciite estreptocócica necrotizante, herpes simples, pioderma gangrenoso, necrose pelo warfarin, dentre outros. ${ }^{2}$

Embora o diagnóstico possa ser feito em bases clínicas na maioria dos pacientes, nos casos de dúvida, a radiografia e ultra-sonografia podem demonstrar a presença de gás e a tomografia é útil não só para fechar o diagnóstico e definir a causa, mas também para demonstrar a extensão da infecção. ${ }^{3}$

O tratamento precoce e agressivo é a única chance de sobrevivência para o paciente. Não tratada ou tratada de maneira tímida e inadequada a Gangrena de Fournier leva à falência progressiva de órgãos e sistemas e ao êxito letal.

\section{6- TRATAMENTO}

O tratamento clássico da Gangrena de Fournier consiste na imediata correção dos distúrbios hidroeletrolíticos, ácido-base e hemodinâmicos, antibioticoterapia de largo espectro e desbridamento cirúrgico de emergência. O objetivo do tratamento cirúrgico é remover todo o tecido necrótico, interromper a progressão do processo infeccioso e minimizar os efeitos tóxicos sistêmicos. ${ }^{20}$

A remoção de todo tecido necrótico nem sempre pode ser feita em apenas um procedimento cirúrgico, exigindo em alguns casos, que o paciente seja operado diversas vezes até o controle completo da infecção. A literatura tem demonstrado que, em média, três ou quatro procedimentos são necessários, porém em casos mais avançados este número pode ultrapassar de 15 procedimentos. ${ }^{2}$

A extensão da ressecção do tecido necrótico, até alcançar tecido viável, deve nortear o cirurgião como objetivo a ser alcançado durante a realização do ato cirúrgico ${ }^{3}$ (Figura 1).

Alguns pacientes irão necessitar procedimentos cirúrgicos complementares ao desbridamento. A colostomia está indicada nas situações que facilitam a contaminação fecal como incontinência anal, destruição esfinctérica pelo processo infeccioso ou perfuração retal., ${ }^{3,29}$ Quando a urina for fator de contaminação está indicada o cateterismo vesical e quando este não é possível, a cistostomia. ${ }^{3}$ 


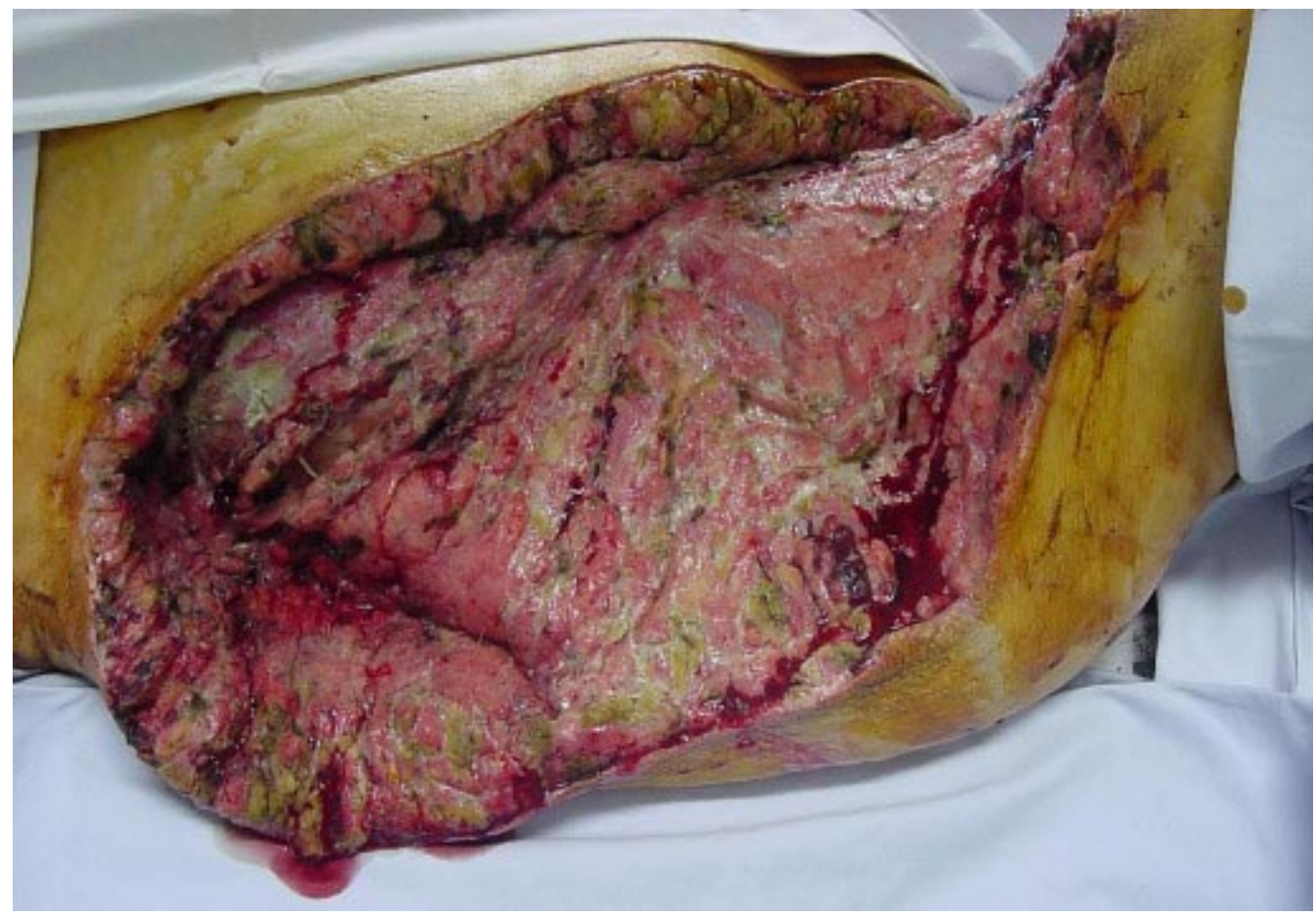

Figura 1: Extensão do desbridamento necessário para o controle da infecção inicialmente localizada no períneo (bartolinite). A foto mostra o paciente em posição de litotomia

Uma decisão crucial no tratamento cirúrgico se apresenta quando os testículos são comprometidos exigindo a orquiectomia. Esse cenário, devido a irrigação testicular independente, embora menos freqüente, não é raro, com incidência de até $21 \%$ em algumas casuísticas ${ }^{2}$. Uma vez constatada a inviabilidade testicular pelo acometimento necrótico o momento e a gravidade da situação não comportam hesitações impondo-se a orquiectomia.

A antibioticoterapia é medida, embora coadjuvante, de fundamental importância no tratamento dos portadores de Gangrena de Fournier. A indicação do antimicrobiano deve responder às questões: qual (ou quais) quando e como.

O tratamento deve ser iniciado assim que o diagnóstico for estabelecido e paralelamente às providências para encaminhar o paciente ao centro cirúrgico.

$\mathrm{O}$ esquema terapêutico deve ser dirigido no sentido de abranger uma flora polimicrobiana e iniciado em bases empíricas, até obter dos resultados obtidos a partir de material enviado para cultura.

Essas circunstâncias, considerando a gravidade da infecção, exigem a adoção de um esquema que propicie cobertura adequada para aeróbios Gram negativos, anaeróbios e estreptococos. ${ }^{9,25}$
Além disso, deve-se considerar a possibilidade da presença de Clostridium tetani e adotar as medidas necessárias para sua prevenção e também a possibilidade de infecção por fungos. O esquema antibiótico mais recomendado é a combinação de penicilinas (penicilina cristalina ou amoxacilina), metronidazol ou clindamicina, e cefalosporina de terceira geração ou aminoglicosídeos. Outras opções, que podem ser utilizados na forma de monoterapia, são as penicilinas com inibidor de 2-lactamase ou carbapenêmicos.

Mesmo instituída a antibioticoterapia, o desbridamento cirúrgico jamais deve ser retardado, devendo ser extenso e repetitivo até se debelar todo o tecido necrótico e ter como limite os tecidos viáveis. Muitas vezes são necessários vários procedimentos cirúrgicos para se controlar o processo de necrose. O sangramento intraoperatório pode ser um fator limitante para que se interrompa a cirurgia.

Os cuidados locais com a ferida, uma vez controlada a infecção também devem ser motivo de atenção. Entre os agentes propostos para esse fim a literatura tem suprido uma vasta relação que abrange substâncias diversas como a colagenase liofilizada (enzima que digere tecido necrótico), carvão ativado, açúcar, mel, papaína, hidróxido de magnésio entre outros. ${ }^{20}$ 
Independente da substância aplicada, a experiência mostra que o principal aspecto relevante no curativo é a limpeza mecânica com soro fisiológico e até com água e sabão neutro.

\section{7- OXIGENOTERAPIA HIPERBÁRICA}

Pelo papel proeminente das bactérias anaeróbicas na fisiopatologia, vários autores recomendam a utilização de oxigenação hiperbárica como um tratamento coadjuvante para esse tipo de infecção. Sugere-se que o uso da terapia hiperbárica pode diminuir a extensão da necrose e reduzir os índices de mortalidade e morbidade. ${ }^{2,3,30}$ Essa terapia tem efeito facilitador na cicatrização de feridas e acelera a recuperação após desbridamento, reduzindo inclusive a necessidade de desbridamentos sucessivos.

A oxigenoterapia hiperbárica exerce um efeito antibacteriano direto sobre os anaeróbios e a atividade de endotoxinas é reduzida na presença de níveis teciduais elevados de oxigênio. Benefícios atribuídos a este tratamento incluem a melhora na ação fagocitária dos neutrófilos, aumento da proliferação dos fibroblastos e da angiogênese, redução do edema devido à vasoconstricção, aumento do transporte intracelular de antibióticos e síntese de radicais livres de oxigênio. Assim, este tratamento está indicado nos pacientes com Gangrena de Fournier que permaneçam toxemia- dos apesar de extenso desbridamento e naqueles com evidência clínica e radiológica de infecção por anaeróbio (Figura 2).

As sessões de oxigenoterapia hiperbárica devem ser diárias com 2 horas de duração com pressão de 2,4 ATA por um período médio de 10 a 15 sesssões dependendo da evolução. Os casos críticos em sepse, internados na UTI, mesmo na vigência de ventilação mecânica e uso de drogas vasoativas, podem ser pressurizados e apresentam benefício com o tratamento. ${ }^{31}$

Com o uso da oxigenoterapia hiperbárica, alguns autores alegam que a mínima remoção de tecidos necróticos e pequenas incisões para drenagem seriam suficientes para controlar o processo, não alterando a sobrevida. ${ }^{32}$

\section{8- FATORES PROGNÓSTICOS}

Mesmo com desbridamento extenso e antibioticoterapia adequada os índices de óbitos permanecem elevados o que levou alguns autores a tentar identificar e validar fatores que possam predispor à maior mortalidade. . $^{817,19,22,28}$

Entretanto, as conclusões desses trabalhos não são consensuais.

Um estudo concluiu que o tempo decorrido entre o início da doença e o tratamento cirúrgico era o fator mais importante na indução de mortalidade. ${ }^{19}$

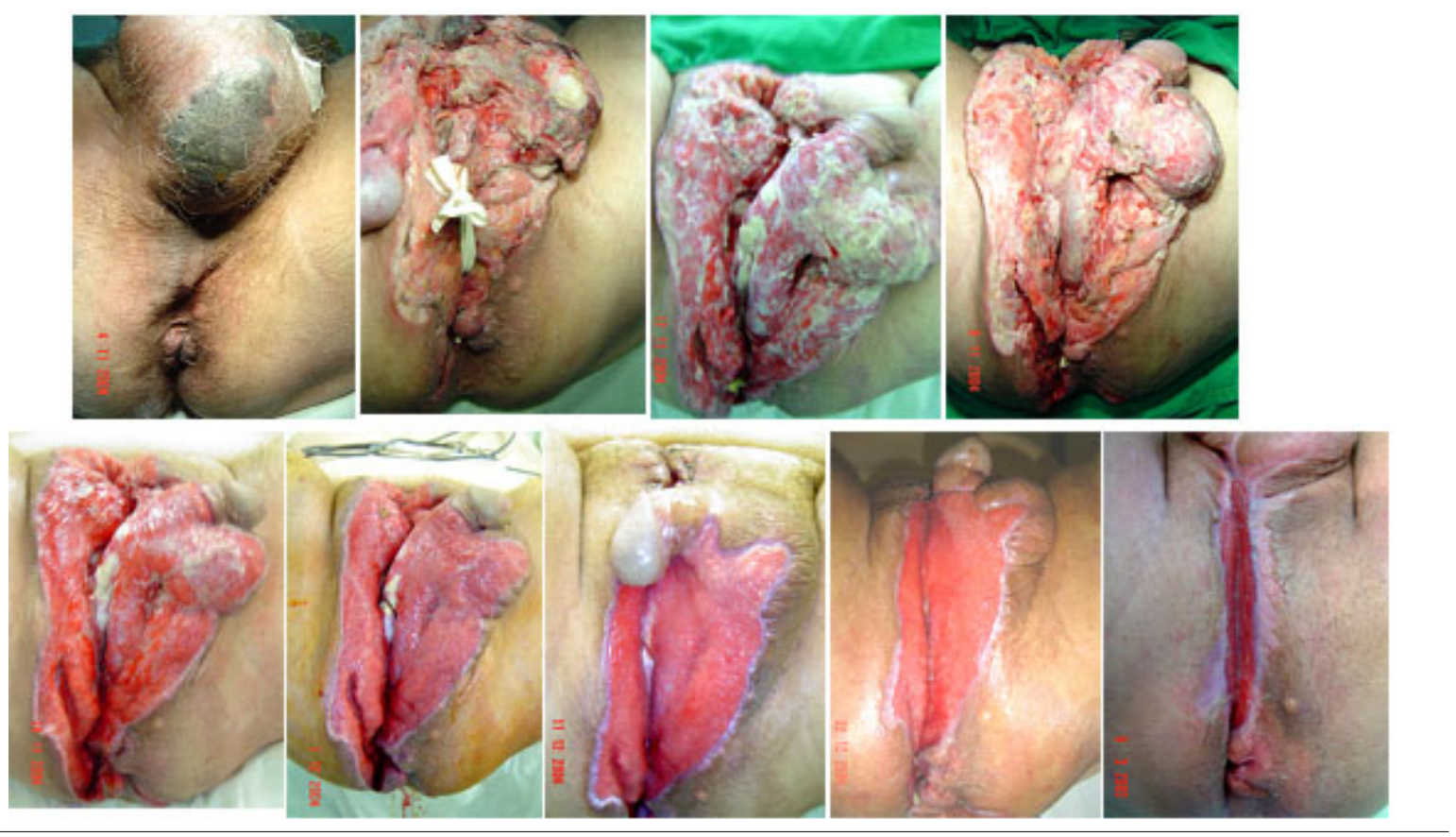

Figura 2: Aspectos evolutivos do tratamento da Gangrena de Fournier mediante tratamento codjuvante com a oxigenioterapia hiperbárica. 
Um outro estudo também conclui que a duração dos sintomas do início do quadro até a internação é um fator contribuinte para a mortalidade e assinala a localização ano retal, a insuficiência renal e a extensão da gangrena como outros fatores importantes. ${ }^{10}$

Correlação entre a extensão da doença e os índices de mortalidade na Gangrena de Fournier foi a conclusão do trabalho de Féres et al. que propuseram uma classificação anatômica que abrange quatro grupos cujas características estão especificada na Tabela II. ${ }^{17}$

Um outro trabalho, analisando vários fatores, pontuando alterações fisiológicas e baseando-se em uma modificação do APACHE II, concluiu que o principal fator influindo na mortalidade era o conjunto de alterações fisiológicas que podem ser agrupadas no que os autores denominaram de Índice de Gravidade da Gangrena de Fournier (IGGF) ${ }^{22}$

Confirmação da validade do IGGF foi estabelecida em dois estudos ${ }^{33,34}$ porém questionada em um terceiro que concluiu que esse índice pode não refletir a gravidade da doença. ${ }^{22}$

Portanto, embora ainda não consensual, os principais fatores relacionados com a mortalidade na Gangrena de Fournier parecem ser: tempo decorrido entre o início da doença e o tratamento cirúrgico, uso precoce da oxigenoterapia hiperbárica ${ }^{32}$ extensão da necrose e repercussões sistêmicas representadas por alterações fisiológicas refletindo o impacto da doença sobre o paciente.

Tabela II: Classificação anatômica da extensão da área de necrose e correlação com mortalidade (Féres et al. 2001) ${ }^{9}$

\begin{tabular}{llc}
\hline Grupos & Descrição & Mortalidade (\%) \\
\hline Grupo I & Necrose do períneo anterior, escroto e pênis ou vulva. & 12,5 \\
Grupo II & Grupo I + períneo posterior, região perianal até 7 cm de diâmetro, & \\
& reto e gordura periretal. & 34 \\
Grupo III & Grupo II + região sacral, glúteo, região inguinal e necrose do pênis. & 37 \\
Grupo IV & $\begin{array}{l}\text { Grupo III + parede abdominal, região suprapúbica, flanco, parede } \\
\text { torácica, região axilar e retro peritônio. }\end{array}$ & 68,75 \\
\hline
\end{tabular}

Cardoso JB, Féres O. Fournier's Gangrene. Medicina (Ribeirão Preto) 2007; 40 (4): 493-9, oct./dec.

ABSTRACT: Fournier's gangrene is a serious infectious disease caused by multiple microorganisms leading to a necrotizing fasciitis of the genitals and perineum. With a rapid and progressive evolution can complicate with sepsis, multiple organ failure and death. Treatment is mainly based in early diagnosis and aggressive and extensive debridment, broadspectrum antibiotics, hyperbaric oxygen therapy, and local wound care are coadjuvants.

Keywords: Fournier Gangrene. Infection; Fasciitis, Necrotizing. Sepsis.

\section{REFERÊNCIAS}

1 - Laucks SS. Fournier's gangrene. Surg Clin North Am 1994; 74: 1339-52.

2 - Smith GL, Bunker CB, Dinneen MD. Fournier's gangrene. Br J Urol 1998; 81: 347-55.
3 - Yaghan RJ, Al-Jaberi TM, Bani-Hani I. Fournier's gangrene. Changing face of the disease. Dis Colon Rectum 2000; 1300-8.

4 - Stephens BJ, Lathrop JC, Rice WT, Gruenberg JC. Fournier's gangrene: historic $(1764-1978)$ versus contemporary (1979 - 1988) differences in etiology and clinical importance. Am Surg 1993; 59: 149-54. 
5 - Fournier JA. Gangrene foudroyante de la verge. Med Prat 1883; 4: 589-97.

6 - Fournier JA Étude clinique de la gangrene foudroyante de la verge. Sem Med 1884; 4: 69.

7 - Yanar H, Taviloglu K, Ertekin C, Guloglu R, Zorba U, Cabioglu N, Baspinar I. Fournier's Gangrene : Risk factors and strategies for management. World J Surg 2006; 30: 1750-4.

8 - Jeong HJ, Park SC, Seo IY, Rim JS. Prognostic factors in Fournier gangrene. Int J Urol 2005; 12: 1041-4

9 - Norton KS, Johnson LW, Perry T, Perry KH, Sehon JK, Zibary GB. Management of Fournier's gangrene: an eleven year retrospective analysis of early recognition, diagnosis, and treatment. Am Surg 2002; 68: 709-13.

10 - Safioleas M, Stamatakos M, Mouxopoulos G, Diaba A, Kontzoglou K, Papachirstodoulou A. Fournier's gangrene: exists and it is still lethal. Int Urol Nephrol 2006; 38: 653-7.

11 - Eke N. Fournier's gangrene: a review of 1726 cases. $\mathrm{Br} \mathrm{J}$ Surg 2000; 87: 718-28.

12 - Spirnak JP, Resnick MI, Hampel N, Persky L. Fournier's gangrene: report of 20 patients. J Urol 1984;131: 289-91.

13 - Uppot RN, Levy HM, Patel PH. Case 54: Fournier Gangrene. Radiology 2003; 226:115-7.

14 - Tran HA, Hart AM. Fournier's gangrene. Intern Med J 2005; 36: 200-1.

15 - Rotondo N. Fournier's gangrene: An unusual presentation of sepsis. J Emerg Med 2002; 23: 413-4.

16 - Lehnhardt M, Steinstraesser L, Druecke D, Muehlberger T, Steinau HU, Homann HH. Fournier's gangrene after milliganmorgan hemorrhoidectomy requiring subsequent abdominoperineal resection of the rectum: report of a case. Dis Colon Rectum 2004; 47: 1729-33.

17 - Féres O, Andrade JI, Rocha JJR, Aprilli F. Fournier's gangrene: a new anatomic classification. In: Reis Neto JA, editor. Proceedings of the 18th Biennial Congress of the International Society of University Colon and Rectal Surgeons; 2000 July 23-26; São Paulo, Brazil. Bologna: Monduzzi Editore; 2000. p. 103-7.

18 - Clayton MD, Fowler JE, Sharifi R. Causes, presentation and survival of 57 patients with necrotizing fasciitis of the male genitalia. Surg Gynaecol Obstet 1990;170: 49-55.

19 - Korkut M, Icoz G, Dayangac M, Akgun E, Yeniay L, Erdogan $\mathrm{O}, \mathrm{Cal} \mathrm{C}$. Outcome analysis in patients with Fournier's gangrene. Report of 45 cases. Dis Colon Rectum 2003; 46: 649-52.

20 - Atakan IH, Kaplan M, Kaya Esat, Aktoz T, Inci O. A lifethreating infection: Fournier's gangrene. Int Urol Nephrol 2002; 34: 387-92.
21 - Nomikos IN. Necrotizing perineal infections (Fournier's disease): old remedies for an old disease. Int $\mathrm{J}$ Colorect Dis 1998; 13: 48-51.

22 - Tuncel A, Aydin O, Tekdogan U, Nalcacioglu V, Capar Y, Atan A. Foournier's gangrene: three years of experience with 20 patiens and validity of the Fournier's gangrene Severity Index Score. Eur Urol 2006; 50: 838-43.

23 - Ochiai T, Ohta K, Takahashi M, Yamazaki S, Iwai T. Fournier's gangrene: report of six cases. Surg Today 2002; 31: 553-6.

24 - McKay TC, Waters WB. Fournier's gangrene as the presenting sign of an undiagnosed human immunodeficiency virus infection. J Urol 1994;152: 1552-4.

25 - Quatan N, Kirby RS. Improving outcomes in Fournier's gangrene. BJU Int 2004; 93: 691-2.

26 - Morpurgo E, Galandiuk S. Fournier's gangrene. Surg Clin Nort Am 2002; 82: 1213-24.

27 - Moreira C, Wongpakdee S, Gennaro A. A foreign body (chicken bone) in the rectum causing perirectal and scrotal abscess. Dis Colon Rectum 1975; 18: 407-9.

28 - Laor E, Palmer LS, Tolia BM, Reid RE Winter HI. Outcome prediction in patients with Fournier's gangrene. J Urol 1995; 154: 89-92.

29 - Murakami M, Okamura K, Hayashi M, Minoh S, Morishige I, Hamano K Fournier's gangrene treated by simultaneously using colostomy and open drainage. J Infect. 2006; 53: 158.

30 - Mindrup SR,Kealey GP, Fallon B. Hyperbaric oxygen for the treatment of Fournier's gangrene. J Urol 2005; 173: 1975-7.

31 - Korhonen K, Hirn M,Niinikoski J. Hyperbaric oxygen in the treatment of Fournier's gangrene. Eur J Surg 1998; 164: 251-5.

32 - Dias MD. Oxigenoterapia hiperbárica em pacientes portadores de lesões teciduais. revisão de 1506 casos. [Tese de Doutorado], São Paulo: Faculdade de Medicina - USP; 2004.

33 - Yeniol CO, Suelozgen T, Arslan M, Ayder AR Fournier's gangrene: experience with 25 patients and use of Fournier's Gangrene Severity Index Score. Urology 2004; 64: 218-22.

34 - Laor E, Palmer LS, Tolia BM, Reid RE Winter HI. Outcome prediction in patients with Fournier's Gangrene. J Urol 1995; 154: 89-92.

Recebido em 29/06/2007

Aprovado em 31/08/2007 